\title{
Soluble urokinase plasminogen activator receptor in one-year prediction of major adverse cardiac events in patients after first myocardial infarction treated with primary percutaneous coronary intervention
}

\author{
Rafał N. Wlazeł로 Marta Migała², Marzenna Zielińska², Lucjan Pawlicki ${ }^{3}$, Kinga Rośniak-Bąk ${ }^{1}$ \\ Iwona Szadkowska
} 1Department of Laboratory Diagnostics and Clinical Biochemistry, Medical University
of Lodz, Lodz, Poland
${ }^{2}$ Department of Intensive Cardiac Therapy, Medical University of Lodz, Lodz, Poland
${ }^{3}$ Department of Internal Diseases and Cardiological Rehabilitation, Medical University
of Lodz, Lodz, Poland

Submitted: 23 May 2016

Accepted: 9 August 2016

Arch Med Sci 2019; 15, 1: 72-77

DOI: https://doi.org/10.5114/aoms.2016.63596

Copyright @ 2016 Termedia \& Banach

\begin{abstract}
Introduction: Soluble urokinase plasminogen activator receptor (sUPAR) level reflects the general condition of the organism and was proved to give independent information in risk stratification of patients. The aim of this study was to assess the usefulness of suPAR in the prediction of adverse cardiac events in patients with first myocardial infarction (MI) undergoing primary percutaneous coronary intervention. Additionally, the diagnostic power of suPAR was assessed.

Material and methods: One hundred and thirty-nine of 150 consecutive patients were included in the study. Serum suPAR level (ELISA, Virogates) as well as C-reactive protein (on admission and at discharge) and maximum troponin $\mathrm{T}$ (assessed from successive 6-hour periods of blood collection) were measured. In the 1-year follow-up study the following major adverse cardiac events were observed: myocardial infarction, revascularization, stroke and death.

Results: Multi-variable analysis revealed prognostic usefulness only for suPAR and glomerular filtration rate: $p<0.0001$ and $p=0.018 ; \mathrm{OR}=2.59$ and $O R=0.98$ respectively, with area under the curve in receiver operating characteristic analysis for both parameters simultaneously $0.89(p<0.0001)$. There was no correlation between suPAR level and the left ventricular dysfunction parameters or the MI type.

Conclusions: Soluble urokinase plasminogen activator receptor level appears to be an independent useful biomarker for the prediction of major adverse cardiac events early after first myocardial infarction. The biomarker's level seems to have more prognostic than diagnostic power.
\end{abstract}

Key words: biomarkers, cardiovascular risk prediction, prognostication after acute myocardial infarction.

\section{Introduction}

Apart from its involvement in hemostasis, soluble urokinase plasminogen activator receptor (sUPAR) is known to play a role in inflammatory

\author{
Corresponding author: \\ Rafal N. Wlazel \\ Department \\ of Laboratory \\ Diagnostics and \\ Clinical Biochemistry \\ Medical University \\ 113 Zeromskiego St \\ 90549 Lodz, Poland \\ Phone: +48 426393655 \\ E-mail: rafal.wlazel@umed. \\ lodz.pl
}


processes. Elevated suPAR levels are associated with infections and pathological conditions, reflecting the probability of worse outcomes [1, 2]. Population studies proved that suPAR levels are related to increased risk of type 2 diabetes mellitus, cardiovascular disease and cancer [3, 4], as elevated suPAR levels reflect subclinical organ damage [5]. Recent studies showed that suPAR is a predictor not only of all-cause mortality in healthy subjects [3] but also mortality associated with major adverse cardiac events (MACE) [6]. For a few years now attention has been paid to the usefulness of sUPAR in cardiovascular diseases including coronary heart disease [7, 8]. However, the prognostic value of sUPAR in patients with acute myocardial infarction (AMI) is still not well known.

The aim of this study was to assess the usefulness of serum sUPAR measurement for the determination of adverse cardiac events in patients with first $\mathrm{AMI}$ undergoing primary percutaneous coronary intervention $(\mathrm{pPCl})$. Soluble urokinase plasminogen activator receptor level was determined next to known biochemical inflammatory and myocardial damage biomarkers as well as left ventricular dysfunction parameters to assess its short-term predictive value and also its diagnostic value.

\section{Material and methods}

One hundred and thirty-nine of 150 consecutive patients with first $\mathrm{AMI}$, treated with $\mathrm{pPCl}$ with stent implantation, were included in this study. The patients were admitted to the Department of Intensive Cardiac Therapy from September 2013 to April 2014. The exclusion criteria were as follows: prior myocardial infarction, prior coronary revascularization, history of heart failure, cirrhosis, connective tissue diseases, coexisting cancer and alcohol abuse. Moreover, 11 cases were excluded from the study because of missing follow-up data. All patients gave written informed consent, and the study was approved by the local bioethics committee.

Peripheral blood samples were obtained on admission and at discharge, 3-5 days after AMI. Baseline concentrations of biochemical and hematological parameters were determined during routine diagnostic procedures, and part of the serum was frozen at $-70^{\circ} \mathrm{C}$ for 5 months to determine suPAR level (ELISA, Virogates). The serum levels of high-sensitive C-reactive protein (hsCRP) (ITA, AU680, Beckman Coulter) and troponin T (TnT) (ECLIA, Cobas e411, Roche Diagnostics) were determined. Additionally, TnT concentration was measured during each successive 6-hour period to determine its highest level. Left ventricular ejection fraction (EF) and wall motion score index (WMSI) were assessed in echocardiography, on the $3^{\text {rd }}$ to $5^{\text {th }}$ day of AMI. During the 1-year follow-up study (follow-up visits every 6-month period or a phone call in case of absence from a follow-up visit), the number of MACE defined as non-fatal $\mathrm{AMI}$, stroke, coronary revascularization and death was observed.

\section{Statistical analysis}

All the results, including baseline characteristics, are presented as mean \pm standard deviation (SD) or mean values with the $5^{\text {th }}$ to $95^{\text {th }}$ percentiles, if necessary, for continuous variables and as percent for categorical variables. The differences between biomarker concentrations were analyzed using the Mann-Whitney test for independent samples and the Wilcoxon test for paired samples. The relation between the suPAR level and baseline characteristics were assessed using Spearman correlation analysis. A comparison of appropriate suPAR, hsCRP and TnT levels between groups, with and without the endpoints, was performed with the Kruskal-Wallis test. Logistic regression models were used for the analysis of the relations between biomarkers determined on admission and/or at discharge and the outcome events during the follow-up period. Forward selection was used, and if patients experienced multiple outcome events, only the first one was included. Additionally, receiver operating characteristic (ROC) curve analysis was performed to determine the predictive value of biomarkers as well as to assign their optimal cut-off values. The statistical analysis was performed using Statistica 10 PL (StatSoft Polska, Krakow, Poland). The significance level of $p<0.05$ was accepted for all the tests.

\section{Results}

The final investigated group consisted of 88 men and 40 women aged between 32 and 85 years, with a median age of 61 (41.1-82.8) years. Eighty-seven $(67 \%)$ patients were diagnosed with ST-elevation $\mathrm{MI}$ and 41 (33\%) patients with non-ST-elevation MI. Only 32 (25\%) patients had an EF below $50 \%$; nevertheless, the median suPAR level in this group of patients did not vary from the median sUPAR level of other patients $(4.7 \mathrm{ng} / \mathrm{ml}$ vs. $4.1 \mathrm{ng} /$ $\mathrm{ml}$ respectively, $p=0.09$ ). Among all 29 MACE observed during the follow-up study, 24 (83\%) events took place during the first 6 months. The numbers of particular end-point events were as follow: 10 non-fatal AMIs, 8 coronary revascularizations, 5 strokes and 6 deaths. Detailed characteristics of the investigated group are presented in Table I.

Among all analyzed parameters, we observed positive correlations only between suPAR level and: sex (female gender $p=0.004, r=0.30$ ), age $(p<0.0001, r=0.32)$, glomerular filtration rate (GFR) ( $p=0.0006, r=0.28)$, and the CRP level measured on admission $(p<0.0001, r=0.36)$ and discharge 
Table I. Detailed characteristics of study group.

\begin{tabular}{|c|c|c|c|}
\hline Parameter & $\begin{array}{l}\text { Patients with } \\
\text { endpoint }\end{array}$ & $\begin{array}{l}\text { Patients without } \\
\text { endpoint }\end{array}$ & $P$-value \\
\hline Gender, women & $15(51.6 \%)$ & $25(17.9 \%)$ & $<0.05$ \\
\hline Age [years] & $65.7 \pm 10.7$ & $59.8 \pm 9.7$ & 0.001 \\
\hline Body mass index $\left[\mathrm{kg} / \mathrm{m}^{2}\right]$ & $27.6 \pm 4.2$ & $27.9 \pm 4.7$ & NS \\
\hline Glomerular filtration rate (CKD-EPI) [ml/min/1.72 m²] & $93 \pm 40$ & $110 \pm 37$ & 0.014 \\
\hline Troponin Tmax [ng/ml] & $3.229 \pm 3.741$ & $2.886 \pm 2.9611$ & NS \\
\hline Ejection fraction \% & $53 \pm 12$ & $55 \pm 9$ & NS \\
\hline C-reactive protein (admission) [mg/l] & $14.1 \pm 21.7$ & $12.5 \pm 33.3$ & 0.02 \\
\hline C-reactive protein (discharge) $[\mathrm{mg} / \mathrm{l}]$ & $43.8 \pm 57.3$ & $29.9 \pm 40.9$ & 0.04 \\
\hline Total cholesterol [mmol/l] & $5.29 \pm 1.37$ & $5.33 \pm 1.08$ & NS \\
\hline Low-density lipoprotein cholesterol [mmol/l] & $3.19 \pm 1.1$ & $3.28 \pm 0.95$ & NS \\
\hline Triglycerides $[\mathrm{mmol} / \mathrm{l}]$ & $1.7 \pm 1.2$ & $1.8 \pm 1.1$ & NS \\
\hline High-density lipoprotein cholesterol [mmol/l] & $1.3 \pm 0.4$ & $1.3 \pm 0.4$ & NS \\
\hline Fibrinogen $[\mathrm{g} / \mathrm{l}]$ & $3.9 \pm 1.8$ & $3.9 \pm 1.8$ & NS \\
\hline Uric acid [ $\mu \mathrm{mol} / \mathrm{l}]$ & $346 \pm 96$ & $317 \pm 89$ & NS \\
\hline
\end{tabular}

CDK-EPI - Chronic Kidney Disease Epidemiology Collaboration, max - maximum concentration, NS - not significant.

$(p=0.0001, r=0.31)$. In the group of patients aged $>65$ years the median SUPAR level was significantly higher in comparison to other patients $(4.9 \mathrm{ng} / \mathrm{ml}$ vs. $4.1 \mathrm{ng} / \mathrm{ml}, p=0.0002)$. The suPAR level was also higher in the cohort of women in comparison to men $(4.9 \mathrm{ng} / \mathrm{ml}$ vs. $4.1 \mathrm{ng} / \mathrm{ml}, p=$ 0.005). There was no correlation between suPAR level and EF, WMSI, or maximum TnT or AMI type $(p>0.05)$. Also no correlation was observed between CRP levels (either measured on admission or at discharge) and the parameters listed above $(p>0.1)$. There was a statistically significant difference in suPAR concentrations in all patients after 1 year $(4.15 \mathrm{ng} / \mathrm{ml}$ and $5.37 \mathrm{ng} / \mathrm{ml}, p<0.0001)$.

We observed statistically significant differences between the sUPAR level in the group of patients who reached the endpoint in comparison with the other patients $(p<000.1)$ : the highest median and lowest values were respectively: $9.21 \mathrm{ng} / \mathrm{ml}, 5.93 \mathrm{ng} / \mathrm{ml}, 3.73 \mathrm{ng} / \mathrm{ml}$ vs. $6.99 \mathrm{ng} / \mathrm{ml}$, $4.06 \mathrm{ng} / \mathrm{ml}, 1.15 \mathrm{ng} / \mathrm{ml} ; p=0.02$. No differences were observed for $\max \operatorname{TnT}(p=0.7)$ or for hsCRP measured at discharge $(p=0.5)$, while the only significance in hsCRP level was observed for the parameter measured on admission (median: 6.8 $\mathrm{mg} / \mathrm{l} \mathrm{vs.} 3.7 \mathrm{mg} / \mathrm{l}, p=0.006)$.

The logistic regression analysis done in a forward step-wise manner revealed that for a suPAR level increase of $1 \mathrm{ng} / \mathrm{ml}$, the OR for developing MACE increases by a factor of $2.8(95 \% \mathrm{Cl}: 1.9-$ 3.9), $\chi^{2}$ statistic 20.3; $p<0.0001$. Among other analyzed parameters only age, sex, GFR and hsCRP measured at discharge appeared to be statistically significant, as presented in Table II. However,

Table II. Results of uni- and multi-variable analysis for prediction of end points

\begin{tabular}{|lccccc|}
\hline Parameter & $P$-value & OR & $95 \% \mathrm{Cl}$ & $\chi^{2}$ & ROC \\
\hline $\begin{array}{l}\text { Uni-variable analysis: } \\
\text { Age }\end{array}$ & 0.0001 & 1.1 & $1.04-1.14$ & 7.7 & 0.73 \\
\hline Gender (women) & 0.005 & 3.3 & $1.4-7.1$ & - & 0.64 \\
\hline C-reactive protein (discharge) & 0.014 & 1.01 & $1.002-1.016$ & 13.2 & 0.62 \\
\hline Glomerular filtration rate & $<0.0001$ & 0.97 & $0.96-0.98$ & 12.9 & 0.75 \\
\hline $\begin{array}{l}\text { Multi-variable analysis: } \\
\text { Soluble urokinase plasminogen activator receptor }\end{array}$ & $<0.0001$ & 2.59 & $1.70-3.95$ & & \\
\hline Glomerular filtration rate & 0.018 & 0.98 & $0.96-0.99$ & & \\
\hline
\end{tabular}


multi-parameter analysis revealed that only suPAR and eGFR contributed significantly to the outcome.

The ROC analysis revealed good predictive value of sUPAR measurement for endpoint prediction in the studied group. The area under the curve (AUC) was 0.83 (95\% Cl: 0.76-0.89), $p<0.0001$. For the best fit suPAR cut-off value $5.25 \mathrm{ng} / \mathrm{ml}$ (criterion corresponding to the highest Youden index) the sensitivity was $70 \%$ and the specificity was $85 \%$. Positive (PPV) and negative (NPV) predictive values, assuming that the ratio of cases in the positive and negative groups reflects the prevalence of outcomes, were $52.6 \%$ and $91.8 \%$ respectively (Figure 1). Additionally, performing ROC analysis for all kinds of endpoints separately, we observed the strongest statistical significance for sUPAR measurement in the prediction of death. The AUC was $0.82(95 \% \mathrm{Cl}$ : 0.75-0.88); $p=0.001$ with sensitivity $83 \%$ and specificity $80 \%$ for the optimal cut-off value $5.3 \mathrm{ng} / \mathrm{ml}$, PPV 14.7\%, NPV $99.1 \%$. The same analysis done for CRP revealed statistical significance in prediction of adverse events in the studied group for CRP measured at discharge (AUC $=0.62, p=0.01$ ) and no statistical significance for CRP measured on admission (AUC $=0.56, p=0.3$ ). These results are in accordance with the correlation analysis and the logistic regression analysis presented above.

\section{Discussion}

Along with new strategies of medical imaging [9], many new biomarkers have recently been studied in the context of their potential usefulness in prediction in patients with cardiovascular disease [10-12]. Among others, such as galectin-3 or GDF15 , suPAR seems to be an important prognostic biomarker in various diseases with an inflammatory response in their pathology, including $\mathrm{MI}$.

In their research, Lyngbaek et al. focused on the usefulness of suPAR in the general (long-term) prediction of repeat $\mathrm{MI}$ and mortality in patients with STEMI treated with the $\mathrm{PCl}$ procedure, obtaining a median endpoint of 5.75 years in follow-up [6]. The authors revealed that suPAR (in contrast to (RP) was a stable plasma biomarker that predicted all-cause mortality and recurrent MI. Our studies are the first to demonstrate that sUPAR adds independent prognostic information also in a population of patients with their first MI, without history of heart failure, successfully treated with $\mathrm{pPCl}$, the majority without significant post-infarction heart muscle damage. The previously mentioned studies assessed the predictive value of suPAR usually in reference only to $\mathrm{MI}$ and mortality, while we focused also on revascularization and stroke and in a relatively short follow-up time. Our results seem to be interesting especially since we also enrolled patients with NSTEMI.
The statistically significant differences in SUPAR levels between patients who finally developed endpoints and other patients, as well as the logistic regression analysis, indicate that processes responsible for adverse events in patients with a first MI may manifest their causes in SUPAR level at discharge, which makes sUPAR a potentially good prediction marker. The cut-off for the best fit AUC in ROC analysis with its value of $5.25 \mathrm{ng} / \mathrm{l}$ is in accordance with data on suPAR cut-off levels associated with higher risk (5.0 males; 5.5 females), based on general population studies [3, 5] followed by information included in the information sheet of the test kit, and recommended by the producer as a high-risk cut-off value. Previously proven stable concentrations of suPAR during the evolution of $\mathrm{MI}$ and coronary surgery $[6,13]$ allow the point of discharge to be a good moment for suPAR testing, which was also confirmed in our studies.

Previous studies have shown that suPAR is an independent predictor of all-cause mortality, not only associated with cardiovascular risk [2, 3, 6, 14]. In our study, despite the small number of deaths observed during the short follow-up, the statistical characteristics of ROC analysis considering SUPAR as a predictor of mortality were the strongest, with $p=0.001$, with the best fit cut-off value of 5.3 .

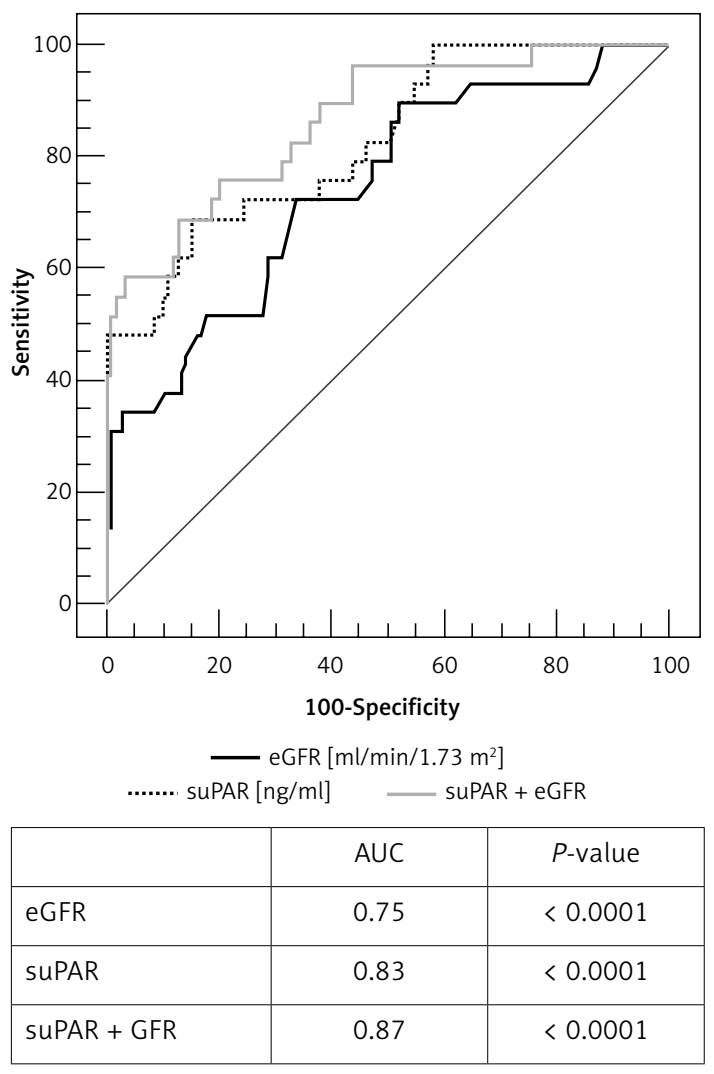

Figure 1. Comparative analysis of ROC curves for prediction of MACE in 1-year follow-up study 
It has also been shown in previous studies that in contrast to SUPAR, the well-known and often used inflammatory biomarker CRP was not, or was only weakly, related to adverse outcomes or mortality [15-17]. In our study all the statistical analysis that was performed for SUPAR was also done for CRP, and it confirmed that in our cohort of patients this biomarker showed neither diagnostic nor prognostic usefulness, with almost all statistical characteristics not significant $(p>0.05)$. This information confirms that the pathophysiology pathways of suPAR in the inflammatory response are different than in CRP, and therefore suPAR provides an independent value of measurement in risk stratification.

This is, to our knowledge, the first study to examine suPAR level, from not only a prognostic but also a diagnostic point of view. We chose myocardial damage biomarkers as well as left ventricular dysfunction parameters to characterize the MI process itself. The main finding was that suPAR level was not correlated with MI type, EF, WMSI or maximum TnT concentration. That may prove that there is no pathophysiological pathway connecting suPAR with myocardial necrosis and its complications and clinical manifestations. On the other hand, these results make the prognostic potential of suPAR stronger.

The question whether SUPAR is more related to adverse outcomes or is an independent marker of low grade inflammation seems to remain unanswered. The last results mentioned in our study prove the growth in SUPAR concentration in a patient's sera during the 1-year follow-up. It may indicate general health deterioration, reflecting vascular dysfunction followed by progressive subclinical organ damage [3], which may lead to a clinical manifestation of acute coronary syndrome in the future. This finding seems to be in accordance with another study performed on an American cohort, describing the relationship of sUPAR with angiographic coronary artery disease [8]. The authors indicated that suPAR levels were lowest in subjects with angiographically normal coronary arteries, compared to those with either insignificant coronary atherosclerosis $(<50 \%$ stenosis) or those with significant coronary artery disease ( $>50 \%$ stenosis) - means of suPAR levels: $3.1 \mathrm{ng} / \mathrm{l}, 3.3 \mathrm{ng} / \mathrm{l}, 3.5 \mathrm{ng} / \mathrm{l}$ respectively. It is also worth mentioning that although the results seem to be promising, the detailed mechanism of release of UPAR from the membrane of particular cells is still unclear.

One review article in its "future work" section pointed out several limitations in studies which consider the usefulness of suPAR [18]. One of them was the question whether SUPAR plays any role in the disease process. The authors also pointed out that the majority of the data is de- rived from the same cohorts from one geographical region (Denmark and Sweden). We hope that our results add new information to that field and contribute to reducing selection bias in the generalization of result findings.

A limitation of our study is the relatively small sample size, which was associated with the fact that not all of the patients met our inclusion and exclusion criteria. Furthermore, the study was conducted in a single hospital only. The biomarker in question should also be correlated with other known biomarkers associated with the inflammatory process, such as procalcitonin, interleukin or complement. The follow-up should be continued.

In conclusion, our results show that the SUPAR level measured on the $3^{\text {rd }}-5^{\text {th }}$ day of $A M I$ is significantly elevated in patients with further major adverse events. The suPAR level assessed at discharge may be a useful independent predictor for cardiovascular events (better than other biomarkers, such as (RP) in patients with the first AMI. Wider use of SUPAR in daily clinical practice needs further investigation.

\section{Acknowledgments}

The study was supported with a scientific grant for statutory activity of the Medical University of Lodz, Poland, no. 503/5-020-01/503-51-001.

\section{Conflict of interest}

The authors declare no conflict of interest.

\section{References}

1. Sidenius N, Sier CF, Ulum H, et al. Serum level of soluble urokinase-type plasminogen activator receptor is a strong and independent predictor of survival in human immunodeficiency virus infection. Blood 2000; 96: 4091-5.

2. Ostrowski SR, Ulum H, Goka BQ, et al. Plasma concentrations of soluble urokinase-type plasminogen activator receptor are increased in patients with malaria and are associated with a poor clinical or a fatal outcome. J Infect Dis 2005; 191: 1331-41.

3. Eugen-Olsen J, Anderson O, Linneberg A, et al. Circulating soluble urokinase plasminogen activator receptor predicts cancer, cardiovascular disease, diabetes and mortality in the general population. J Intern Med 2010; 268: 296-308.

4. De Bock CE, Wang Y. Clinical significance of urokinase-type plasminogen activator receptor (UPAR) expression in cancer. Med Res Rev; 24: 13-39.

5. Sehestedt T, Lyngbaek S, Eugen-Olsen J, et al. Soluble urokinase plasminogen activator receptor is associated with subclinical organ damage and cardiovascular events. Atherosclerosis 2011; 216: 237-43.

6. Lyngbaek S, Marott JL, Moler DV, et al. Usefulness of soluble urokinase plasminogen activator receptor to predict repeat myocardial infarction and mortality in patients with ST-segment elevation myocardial infarc- 
tion undergoing primary percutaneous intervention. Am J Cardiol 2012; 110: 1756-63.

7. Lyngbaek S, Adersson C, Marott IL, et al. Soluble urokinase plasminogen activator receptor for risk prediction in patients admitted with acute chest pain. Clin Chem 2013; 59: 1621-9.

8. Eapen DJ, Manocha P, Ghasemzedah N, et al. Soluble urokinase plasminogen activator receptor level is an independent predictor of the presence and severity of coronary artery disease and of future adverse events. J Am Heart Assoc 2014; 3: e001118.

9. Liszka J, Haberka M, Tabor Z, Finik M, Gąsior Z. Two-dimensional speckle-tracking echocardiography assessment of left ventricular remodeling in patients after myocardial infarction and primary reperfusion. Arch Med Sci 2014; 10: 1091-100.

10. Szadkowska I, Wlazeł RN, Migała M, et al. The association between galectin-3 and clinical parameters in patients with first acute myocardial infarction treated with primary percutaneous coronary angioplasty. Cardiol J 2013; 20: 577-82.

11. Szadkowska I, Wlazel RN, Migala M, et al. The association between galectin-3 and occurrence of reinfarction early after first myocardial infarction treated invasively. Biomarkers 2013; 18: 655-9.

12. Ding Y, Gao BB, Zhou L, et al. Clinical implications of plasma Nogo-A levels in patients with coronary heart disease. Arch Med Sci 2017; 13: 771-7.

13. Gozdzik W, Adamik B, Gozdzik A, Rachwalik M, Kustrzycki W, Kubler A. Unchanged plasma levels of the soluble urokinase-type plasminogen activator receptor in elective coronary artery bypass graft surgery patients and cardiopulmonary bypass use. PLoS One 2014; 9: e98923.

14. Koch A, Tacke F. Why high suPAR is not super - diagnostic, prognostic and potential pathogenic properties of a novel biomarker in the ICU. Crit Care 2011; 15: 1020.

15. Lyngbaek S, Sehestedt T, Marott JL, et al. CRP and suPAR are differently related to anthropometry and subclinical organ damage. Int I Cardiol 2013; 167: 781-5.

16. Lyngbaek S, Marott JL, Sehestedt T, et al. Cardiovascular risk prediction in the general population with use of sUPAR, CRP and Framingham Risk Score. Int J Cardiol 2013; 167: 2904-11.

17. Sorensen MH, Gerke O, Eugen-Olsen J, et al. Soluble urokinase plasminogen receptor is in contrast to high-sensitive C-reactive-protein associated with coronary artery calcification in healthy middle-aged subjects. Atherosclerosis 2014; 237: 60-6.

18. Hodges GW, Bang CN, Wachtell K, Eugen-Olsen J, Jeppesen JL. SUPAR: a new biomarker for cardiovascular disease? Can J Cardiol 2015; 31: 1293-302. 